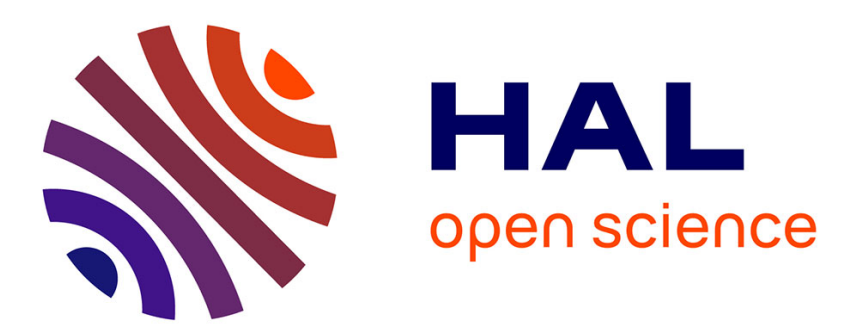

\title{
A general multi-scale two-level optimisation strategy for designing composite stiffened panels
}

\author{
Marco Montemurro, Alfonso Pagani, Giacinto Alberto Fiordilino, Jérôme \\ Pailhès, Erasmo Carrera
}

\section{- To cite this version:}

Marco Montemurro, Alfonso Pagani, Giacinto Alberto Fiordilino, Jérôme Pailhès, Erasmo Carrera. A general multi-scale two-level optimisation strategy for designing composite stiffened panels. Composite Structures, 2018, 201, pp.968-979. 10.1016/j.compstruct.2018.06.119 . hal-02354568

\section{HAL Id: hal-02354568 \\ https://hal.science/hal-02354568}

Submitted on 25 Nov 2019

HAL is a multi-disciplinary open access archive for the deposit and dissemination of scientific research documents, whether they are published or not. The documents may come from teaching and research institutions in France or abroad, or from public or private research centers.
L'archive ouverte pluridisciplinaire HAL, est destinée au dépôt et à la diffusion de documents scientifiques de niveau recherche, publiés ou non, émanant des établissements d'enseignement et de recherche français ou étrangers, des laboratoires publics ou privés. 


\title{
A general multi-scale two-level optimisation strategy for designing composite stiffened panels
}

\author{
Marco Montemurro ${ }^{\mathrm{a}, *}$, Alfonso Pagani ${ }^{\mathrm{b}}$, Giacinto Alberto Fiordilino ${ }^{\mathrm{a}, \mathrm{b}}$, Jérôme Pailhès ${ }^{\mathrm{a}}$, \\ Erasmo Carrerab \\ ${ }^{a}$ Arts et Métiers ParisTech, I2M CNRS UMR 5295, F-33400 Talence, France \\ ${ }^{b}$ MUL2 group, DIMEAS, Politecnico di Torino, Torino, Italy
}

\begin{abstract}
This work deals with the problem of the least-weight design of a composite stiffened panel subject to constraints of different nature (mechanical, geometrical and manufacturability requirements). To face this problem, a multi-scale two-level (MS2L) design methodology is proposed. This approach aims at optimising simultaneously both geometrical and mechanical parameters for skin and stiffeners at each characteristic scale (mesoscopic and macroscopic ones). In this background, at the first level (macroscopic scale) the goal is to find the optimum value of geometric and mechanical design variables of the panel minimising its mass and meeting the set of imposed constraints. The second-level problem focuses on the laminate mesoscopic scale and aims at finding at least one stacking sequence (for each laminate composing the panel) meeting the geometrical and material parameters provided by the first-level problem. The MS2L optimisation approach is based on the polar formalism to describe the macroscopic behaviour of the composites and on a special genetic algorithm to perform optimisation calculations. The quality of the optimum configurations is investigated, a posteriori, through a refined finite element model of the stiffened panel making use of elements with different kinematics and accuracy in the framework of the Carrera's Unified Formulation (CUF).
\end{abstract}

Keywords: Composites, Finite Element Method, Buckling, Optimisation, Lightweight structures.

This is a pre-print of an article published in Composite Structures.

The final authenticated version is available online at:

https://doi.org/10.1016/j. compstruct.2018.06.119

\footnotetext{
*Corresponding author. Tel.: +33 556845 422, Fax.: +335400 06964.

Email address: marco.montemurro@ensam.eu, marco.montemurro@u-bordeaux.fr (Marco Montemurro)
} 


\section{Introduction}

Anisotropic materials, such as fibres-reinforced composites, are extensively used in many industrial fields thanks to their peculiar features: high stiffness-to-weight and strengthto-weight ratios that lead to a substantial weight saving when compared to metallic alloys.

The problem of designing a composite structure is quite cumbersome and can be considered as a multi-scale optimisation problem. The complexity of the design process is actually due to two intrinsic properties of composite materials, i.e., heterogeneity and anisotropy. Heterogeneity gets involved mainly at the microscopic scale (i.e., that of constitutive phases), whilst anisotropy intervenes at both mesoscopic scale (that of the constitutive lamina) and macroscopic one (that of the laminate).

To illustrate the difficulty of properly design/optimise at each relevant scale a composite structure the study presented in this work focuses on a real-world engineering problem that can be considered as paradigmatic: the multi-scale design of a least-weight composite stiffened panel subject to a given set of constraints of different nature (geometrical, mechanical, technological, etc.).

Stiffened panels are widely used in many structural applications, mostly because they allow for a substantial weight saving. Of course, this point is of paramount importance especially in aircraft design, where an important reduction of the structural mass can be achieved if composite laminates are used in place of aluminium alloys. A drawback of such a choice is that the design process becomes more cumbersome than that of a classical metallic structure. In fact, though the use of laminated structures is not a recent achievement in structural mechanics, up to now no general methods exist for their optimum design. In practical applications, engineers always use some simplifying rules to take into account for some relevant properties (which are very difficult to be formalised otherwise).

Several works on the optimum design of composite stiffened panels can be found in literature. Nagendra et al. [1] made use of a standard genetic algorithm (GA) to find a solution for the problem of minimising the mass of a composite stiffened panel subject to constraints on the first buckling load, on maximum allowable strains and "technological" constraints on ply orientation angles. In [2] Bisagni and Lanzi defined a single-step postbuckling optimisation procedure for the design of composite stiffened panels subjected to compression load. The procedure was based on a global approximation strategy, where the structure response is given by an artificial neural network (ANN) trained by means of finite element (FE) analyses, while the optimisation tool consisted in a standard GA. Lanzi and Giavotto [3] proposed a multi-objective optimisation procedure for the design of composite stiffened panels capable to take into account the post-buckling behaviour. The procedure made use of a standard GA and three different methods for surrogate modelling: ANN, Radial Basis Functions and Kriging approximation. In [4] Barkanov et al. dealt with the problem of the optimum design of lateral wing upper covers by considering different kinds of stiffeners and loading conditions. Liu et al. [5] utilised the smeared stiffness-based method for finding the best stacking sequences of composite wings with blending and manufacturing constraints by considering a set of pre-defined fibre angles, i.e., $0^{\circ}, 90^{\circ}$ and $\pm 45^{\circ}$. In [6] López et al. proposed a deterministic and reliability-based design optimisation of composite stiffened panels considering post-buckling regime and a progressive failure analysis. Further works on this topic can be found in literature. For example, and without any ambition of exhaustiveness, the studies of Lillico et al. [7], Butler and Williams [8], Wiggenraad et al. [9], Kaletta and Wolf [10] can be cited too.

A common limitation of the previous works is the utilisation of simplifying hypotheses and rules in the formulation of the stiffened panel design problem. These restrictions mainly focus on the nature of the stacking sequence of the laminates constituting the panel. 
These assumptions are used on the one hand to obtain a short-cut to a possible solution, i.e., to eliminate from the true problem some particularly difficult points or properties to be obtained. On the other hand, some of such rules are considered to prevent the final structure from some undesired phenomena, though this is never clearly and rigorously stated and proved. Unfortunately, the use of these simple rules has a main drawback: the design space is extremely shrunk, thus their utilisation automatically drives the optimisation algorithm only towards suboptimal solutions.

Two examples are the use of symmetric stacking sequences, a sufficient but not necessary condition for membrane-bending uncoupling and the use of balanced stacks to obtain orthotropic laminates. When symmetric stacks are utilised, the design is done using half of the layers, which means also half of the design variables. This kind of stack implicitly implies a reduction of the design space: it is very difficult to obtain the lightest structure under this hypothesis. Conversely, the use of balanced stacks, a sufficient condition for membrane orthotropy, leads systematically to misleading solutions: whenever such a rule is used, bending orthotropy, a rather difficult property to be obtained [11], is simply understated, assumed, but not really obtained, as in [12-15].

In aircraft structural design, some other rules are imposed to the design of composite stiffened panels, although some of them are not mechanically well justified, see for instance $[12,15]$. Among these rules, the most significant restriction is represented by the utilisation of a limited set of values for the layers orientation angles which are often limited to the canonical values of $0^{\circ}, 90^{\circ}$ and $\pm 45^{\circ}$.

To overcome the previous restrictions, in the present study the multi-scale two-level (MS2L) optimisation approach for designing anisotropic complex structures [16-18] is utilised in the framework of the multi-scale optimisation of composite stiffened panels. The proposed MS2L approach aims at proposing a very general formulation of design problem without introducing simplifying hypotheses and by considering, as design variables, the full set of geometric and mechanical parameters defining the behaviour of the panel at each characteristic scale (mesoscopic and macroscopic).

In the context of the MS2L methodology, the optimisation problem is split in two distinct (but related) sub-problems. At the first level (macroscopic scale) the goal is to find the optimum value of geometric and mechanical design variables of the panel minimising its mass and meeting the set of imposed constraints. The second-level problem focuses on the laminate mesoscopic scale (i.e., the ply-level) and aims at finding at least one optimum stack (for each laminate composing the panel) meeting the geometrical and material parameters resulting from the first-level problem. The MS2L approach is based on the utilisation of the polar formalism [19] as well as on a GA previously developed by the first author [20].

The quality of the optimum configurations is investigated, a posteriori, through a refined finite element model of the stiffened panel making use of elements with different kinematics and accuracy (in a global-local sense) in the framework of the Carrera's Unified Formulation (CUF).

The paper is organised as follows: the design problem as well as the MS2L optimisation strategy are discussed in Section 2. The mathematical formulation of the first-level problem is detailed in Section 3, while the problem of determining a suitable laminate stacking sequence is formulated in Section 4. A concise description of the Finite Element (FE) models of the stiffened panel are given in Section 5, while the numerical results of the optimisation procedure are shown in Section 6. Finally, Section 7 ends the paper with some concluding remarks. 


\section{Multi-scale optimisation of composite stiffened panels}

\subsection{Problem Description}

The optimisation strategy presented in this study is applied to the repetitive unit (RU) of a composite stiffened panel typically utilised in aircraft wings. The RU is composed by the union of a skin and a "omega" shaped stringer (or stiffener) as illustrated in Fig. 1. The overall size of the RU are fixed: $a=150 \mathrm{~mm}$ is the width of the RU, while $b=600 \mathrm{~mm}$ is its length which represents also the distance between two consecutive ribs. It must be noted that stiffeners are equispaced over the panel with a step length equal to $a$. Both skin and stiffener are made of carbon-epoxy unidirectional orthotropic laminae whose properties are listed in Table 1 (taken from [11, 21, 22]).

The fundamental hypotheses about the macroscopic mechanical response of the RU focus essentially on the laminate behaviour and geometry (for both skin and stringer).

- Each laminate is made of identical plies (i.e., same thickness $t_{\mathrm{ply}}$ and material).

- The material of the constitutive layer has a linear elastic transverse isotropic behaviour.

- Each laminate is quasi-homogeneous and fully orthotropic [18, 22-24].

- At the macroscopic scale the elastic response of each laminate is described in the theoretical framework of the FSDT and the stiffness matrices of the plate are expressed in terms of the laminate polar parameters [11,21].

- No delamination occurs at the plies interface for both skin and stringer [25].

It is noteworthy that, no simplifying hypotheses are made on the geometric and mechanical parameters of the RU (e.g., on the nature of the stacking sequences). Only avoiding the utilisation of a priori assumptions that extremely shrink the solution space (e.g., the utilisation of symmetric, balanced stacks to attain membrane/bending uncoupling and membrane orthotropy, respectively) one can hope to obtain the best optimum solution for a given problem: this is a key-point in the proposed approach.

\subsection{Description of the multi-scale two-level optimisation strategy}

The main goal of the MS2L optimisation strategy is the least-weight design of the composite stiffened panel subject to constraints of different nature, i.e., mechanical, geometrical as well as feasibility and technological requirements. The optimisation procedure is articulated into the following two distinct (but related) optimisation problems.

First-level problem. The aim of this phase is the determination of the optimal value of both mechanical and geometric parameters of the laminate composing the RU of the panel in order to minimise its weight and to satisfy, simultaneously, the full set of imposed requirements (formulated as optimisation constraints). At this level each laminate is modelled as an equivalent homogeneous anisotropic plate whose behaviour is described in terms of the laminate polar parameters [11,21]. Therefore, the design variables of this phase are the geometric parameters of the RU as well as the laminate polar parameters of both skin and stiffener.

Second-level problem. The second level of the strategy aims at determining a suitable lay-up for both skin and stringer laminates (i.e., the laminate mesoscopic scale) meeting the optimum combination of their material and geometrical parameters provided by the first-level problem. The goal is, hence, to find at least one stacking sequence (for each 
laminate) which has to be quasi-homogeneous, fully orthotropic and that has to satisfy the optimum values of the polar parameters resulting from the first step. At this level of the strategy, the design variables are the layer orientations.

\section{Mathematical formulation of the first-level problem}

The overall features of the structure at the macroscopic scale have to be optimised during this phase. The mass minimisation of the stiffened panel RU will be performed by satisfying the set of optimisation constraints listed below:

1. a constraint on the first buckling load of the RU;

2. geometric and technological constraints related to the geometrical parameters of the $\mathrm{RU}$;

3. feasibility constraints on the laminate polar parameters of both skin and stringer.

These aspects are detailed in the following subsections.

\subsection{Geometrical design variables}

The design variables for the problem at hand are of two types: geometrical and mechanical. Some of the geometrical parameters of the RU of the stiffened panel are illustrated in Fig. 1. Of course, these parameters are not independent. The independent geometric design variables are:

- the laminate thickness for both skin and stringer, i.e., $t_{S}$ and $t_{B}$, respectively;

- the width $a_{2}$ of the stringer bottom flange;

- the stringer height $h$;

- the size $a_{3}$.

The size $a_{1}$ can be related to the previous variables,

$$
a_{1}=\frac{a}{2}-a_{2}-a_{3},
$$

while the angle of the inclined wall of the stiffener is

$$
\theta=\operatorname{atan}\left(\frac{h}{a_{3}-\frac{a_{2}}{2}}\right) .
$$

The previous design variables must satisfy a set of technological and geometrical requirements. Firstly, the overall thickness of the laminates composing the $\mathrm{RU}$ is a discrete variable, the discretisation step being equal to the thickness of the elementary layer, i.e., $t_{\text {ply }}$ (see Table 1 ):

$$
t_{\alpha}=n_{\alpha} t_{\mathrm{ply}}, \quad \alpha=S, B,
$$

where $n_{S}$ and $n_{B}$ are the number of layers of skin and stiffener, respectively. It must be highlighted that the optimum value of the laminate thickness determines also the optimum 
number of layers $n$ to be used during the second-level design problem (for both skin and stringer). Secondly, parameters $a_{i},(i=1,2,3)$ have to meet the following conditions:

$$
\begin{aligned}
& a_{1}>0, \\
& a_{3} \geq \frac{a_{2}}{2} .
\end{aligned}
$$

First inequality is necessary to avoid contact between two consecutive stringers, while second one must be imposed in order to keep $\theta$ non-negative. In the framework of the mathematical formalisation of the first-level problem, it is useful to introduce dimensionless geometric design variables, as follows:

$$
c_{1}=2 \frac{a_{2}}{a}, c_{2}=2 \frac{a_{3}}{a_{2}}, c_{3}=\frac{h}{a_{2}} .
$$

The dimensionless geometric parameters can be collected into the vector of geometric design variables defined as:

$$
\boldsymbol{\xi}_{\boldsymbol{g}}^{\mathrm{T}}=\left\{n_{S}, n_{B}, c_{1}, c_{2}, c_{3}\right\}
$$

In this background, inequalities of Eq. (4) can be reformulated as:

$$
\begin{aligned}
& g_{1}\left(\boldsymbol{\xi}_{\boldsymbol{g}}\right)=2 c_{1}+c_{1} c_{2}-2<0, \\
& g_{2}\left(\boldsymbol{\xi}_{\boldsymbol{g}}\right)=1-c_{2} \leq 0 .
\end{aligned}
$$

\subsection{Mechanical design variables}

In the framework of the FSDT [26] the constitutive law of the laminate (expressed within its global frame $R=\{0 ; x, y, z\})$ can be stated as:

$$
\begin{aligned}
& \left\{\begin{array}{l}
\mathrm{N} \\
\mathrm{M}
\end{array}\right\}=\left[\begin{array}{ll}
\mathrm{A} & \mathrm{B} \\
\mathrm{B} & \mathrm{D}
\end{array}\right]\left\{\begin{array}{l}
\varepsilon_{0} \\
\chi_{0}
\end{array}\right\}, \\
& \mathbf{F}=\mathbf{H} \gamma_{0},
\end{aligned}
$$

where $\mathbf{A}, \mathbf{B}$ and $\mathbf{D}$ are the membrane, membrane/bending coupling and bending stiffness matrices of the laminate, while $\mathbf{H}$ is the out-of-plane shear stiffness matrix. $\mathbf{N}, \mathbf{M}$ and $\mathbf{F}$ are the vectors of membrane forces, bending moments and shear forces per unit length, respectively, whilst $\varepsilon_{0}, \chi_{0}$ and $\gamma_{0}$ are the vectors of in-plane strains, curvatures and outof-plane shear strains of the laminate middle plane, respectively, (in the previous equations Voigt's notation has been utilised [26]).

In order to analyse the elastic response of the multilayer structure the best practice 
consists in introducing the laminate normalised stiffness matrices:

$$
\begin{aligned}
& \mathbf{A}^{*}=\frac{1}{t} \mathbf{A}, \\
& \mathbf{B}^{*}=\frac{2}{t^{2}} \mathbf{B}, \\
& \mathbf{D}^{*}=\frac{12}{t^{3}} \mathbf{D}, \\
& \mathbf{H}^{*}= \begin{cases}\frac{1}{t} \mathbf{H} & \text { (basic), } \\
\frac{12}{5 t} \mathbf{H} & \text { (modified). }\end{cases}
\end{aligned}
$$

where $t$ is the total thickness of the laminate.

As discussed in [11, 21], in the framework of the polar formalism it is possible to express the Cartesian components of these matrices in terms of their elastic invariants. It can be proven that, in the FSDT framework, for a fully orthotropic, quasi-homogeneous laminate (i.e., a laminate having the same orthotropic behaviour in terms of normalised membrane and bending stiffness matrices and whose membrane/bending coupling stiffness matrix is null) the overall number of independent mechanical design variables describing its mechanical response reduces to only three, i.e., the anisotropic polar parameters $R_{0 K}^{A^{*}}$ and $R_{1}^{A^{*}}$ and the polar angle $\Phi_{1}^{A^{*}}$ (this last representing the orientation of the main orthotropy axis) of matrix $\mathbf{A}^{*}$. For more details on the polar formalism and its application in the context of the FSDT the reader is addressed to [11, 21, 27].

In addition, in the formulation of the optimisation problem for the first level of the strategy, the feasibility constraints on the polar parameters (which arise from the combination of the layers orientations and positions within the stack) must also be considered. These constraints ensure that the optimum values of the polar parameters resulting from the first step correspond to a feasible laminate that will be designed during the second step of the MS2L strategy, see [28]. Since the laminate is quasi-homogeneous, such constraints can be written only for matrix $\mathbf{A}^{*}$ :

$$
\left\{\begin{array}{c}
-R_{0} \leq R_{0 K}^{A^{*}} \leq R_{0}, \\
0 \leq R_{1}^{A^{*}} \leq R_{1} \\
2\left(\frac{R_{1}^{A^{*}}}{R_{1}}\right)^{2}-1-\frac{R_{0 K}^{A^{*}}}{R_{0}} \leq 0 .
\end{array}\right.
$$

In Eq. (11), $R_{0}$ and $R_{1}$ are the anisotropic moduli of the ply reduced stiffness matrix [11]. As in the case of geometric design variables, it is very useful to introduce the following dimensionless quantities:

$$
\rho_{0}=\frac{R_{0 K}^{A^{*}}}{R_{0}}, \rho_{1}=\frac{R_{1}^{A^{*}}}{R_{1}} .
$$

In this background, Eq. (11) writes:

$$
\left\{\begin{array}{c}
-1 \leq \rho_{0} \leq 1 \\
0 \leq \rho_{1} \leq 1 \\
2\left(\rho_{1}\right)^{2}-1-\rho_{0} \leq 0 .
\end{array}\right.
$$


The mechanical design variables must be considered for each laminate constituting the panel RU, i.e., for both skin and stiffener laminates $\left(\rho_{0 \alpha}\right.$ and $\rho_{1 \alpha}$ with $\left.\alpha=S, B\right)$. Moreover, the main orthotropy direction of the generic laminate can be set equal to zero, i.e., $\Phi_{1}^{A^{*}}=0$ for skin and stringer, which means that the main orthotropy axis is aligned with the direction of the applied load. Therefore, the dimensionless mechanical parameters defined above can be grouped into the vector of mechanical design variables:

$$
\boldsymbol{\xi}_{m}^{\mathrm{T}}=\left\{\rho_{0 S}, \rho_{1 S}, \rho_{0 B}, \rho_{1 B}\right\} .
$$

First and second constraints of Eq. (13) can be taken into account as admissible intervals for the relevant optimisation variables, i.e., on $\rho_{0}$ and $\rho_{1}$. Hence, the resulting feasibility constraints on the skin and stringer dimensionless polar parameters become:

$$
\begin{aligned}
& g_{3}\left(\boldsymbol{\xi}_{\boldsymbol{m}}\right)=2\left(\rho_{1 S}\right)^{2}-1-\rho_{0 S} \leq 0, \\
& g_{4}\left(\boldsymbol{\xi}_{\boldsymbol{m}}\right)=2\left(\rho_{1 B}\right)^{2}-1-\rho_{0 B} \leq 0 .
\end{aligned}
$$

For a wide discussion upon the laminate feasibility and geometrical bounds as well as on the importance of the quasi-homogeneity assumption the reader is addressed to [28].

\subsection{Mathematical statement of the problem}

As previously stated, the aim of the first-level optimisation is the minimisation of the mass of the RU of the stiffened panel by satisfying, simultaneously, constraints of different nature. The design variables (both geometrical and mechanical) of the problem can be collected into the following vector:

$$
\boldsymbol{\xi}^{T}=\left\{\boldsymbol{\xi}_{\boldsymbol{g}}^{T}, \boldsymbol{\xi}_{\boldsymbol{m}}^{T}\right\}
$$

In this context the optimisation problem can be formulated as a classical constrained nonlinear programming problem (CNLPP):

$$
\begin{aligned}
& \min _{\boldsymbol{\xi}} \frac{M(\boldsymbol{\xi})}{M_{\text {ref }}} \\
& \text { subject to: } \\
& \left\{\begin{array}{l}
1.05-\frac{\lambda(\boldsymbol{\xi})}{\lambda_{\text {ref }}} \leq 0, \\
g_{i}(\boldsymbol{\xi}) \leq 0, \text { with } i=1, \cdots, 4 .
\end{array}\right.
\end{aligned}
$$

The design space of the first-level problem, together with the type of each design variable, is detailed in Table 2. In Eq. (17) $M$ is the overall mass of the RU, $\lambda$ is the first buckling load of the stiffened panel, while $M_{\text {ref }}$ and $\lambda_{\text {ref }}$ are the counterparts for a reference solution which is subject to the same boundary conditions (BCs) as those applied on the RU of the panel that will be optimised. The properties of the reference configuration of the RU are reported in Table 3.

\subsection{Numerical strategy}

Problem (17) is a non-convex CNLPP in terms of both geometrical and mechanical variables. Its non-linearity and non-convexity is due on the nature of the buckling load 
constraint that is a non-convex function. In addition, the complexity of such a problem is also due to the non-linear feasibility constraints on the laminate polar parameters.

The total number of design variables is nine while that of optimisation constraints is five (see Eq. (17)). Furthermore, the nature of design variables is different (see Table 2): integer $\left(n_{S}\right.$ and $\left.n_{B}\right)$, discrete $\left(c_{1}, c_{2}, c_{3}\right)$ and continuous $\left(\rho_{0 S}, \rho_{1 S}, \rho_{0 B}, \rho_{1 B}\right)$ variables are involved in the definition of this CNLPP.

For the resolution of problem (17) the GA BIANCA [20, 29] coupled with the FE model of the panel RU (for calculating the first buckling load of the structure) has been utilised as optimisation tool for the solution search, see Fig. 2. The GA BIANCA was already successfully applied to solve different kinds of real-world engineering problems, see for example [30-33].

As shown in Fig. 2, for each individual at each generation, the numerical tool performs a FE analysis for calculating the first buckling load (eigenvalue problem) of the stiffened panel as well as its weight. The inputs of the FE model of the RU (implemented in ANSYS ${ }^{\circledR}$ environment) are both geometrical and mechanical parameters (generated by BIANCA). The GA elaborates the results provided by the FE model in order to execute the genetic operations. These operations are repeated until the GA meets the user-defined convergence criterion.

The generic individual (i.e., a generic point in the design space) of the GA BIANCA represents a potential solution for the problem at hand. The genotype of the individual for problem (17) is characterised by only one chromosome composed of nine genes, each one coding a component of the vector of design variables, see Eq. (16).

\section{Mathematical formulation of the second-level problem}

The second-level problem focuses on the lay-up design of the both skin and stringer laminates. The goal is to determine at least one stacking sequence satisfying the optimum values of both geometric and polar parameters resulting from the first level of the strategy and having the elastic symmetries imposed to the laminate within the formulation of the first-level problem, i.e., quasi-homogeneity and orthotropy. In the framework of the FSDT and considering the polar formalism for representing the laminate stiffness matrices, this problem can be stated in the form of an unconstrained minimisation problem [11, 21]:

$$
\min _{\boldsymbol{\delta}} I\left(f_{i}(\boldsymbol{\delta})\right)
$$

with

$$
I\left(f_{i}(\boldsymbol{\delta})\right)=\sum_{i=1}^{6} f_{i}(\boldsymbol{\delta}) .
$$

where $\delta \in \mathbb{R}^{n}$ is the vector of the layer orientations, i.e., the design variables of this phase, while $f_{i}(\boldsymbol{\delta})$ are quadratic functions in the space of polar parameters, each one representing a requirement to be satisfied, such as orthotropy, uncoupling, etc. For the problem at hand 
the partial objective functions are:

$$
\begin{gathered}
f_{1}(\boldsymbol{\delta})=\left(\frac{\left|\Phi_{0}^{A^{*}}(\boldsymbol{\delta})-\Phi_{1}^{A^{*}}(\boldsymbol{\delta})\right|}{\pi / 4}-K^{A^{*}(o p t)}\right)^{2}, \quad f_{2}(\boldsymbol{\delta})=\left(\frac{R_{0}^{A^{*}}(\boldsymbol{\delta})-R_{0}^{A^{*}(o p t)}}{R_{0}}\right)^{2}, \\
f_{3}(\boldsymbol{\delta})=\left(\frac{R_{1}^{A^{*}}(\boldsymbol{\delta})-R_{1}^{A^{*}(o p t)}}{R_{1}}\right)^{2}, \quad f_{4}(\boldsymbol{\delta})=\left(\frac{\left|\Phi_{1}^{A^{*}}(\boldsymbol{\delta})-\Phi_{1}^{A^{*}(o p t)}\right|}{\pi / 4}\right)^{2}, \quad f_{5}(\boldsymbol{\delta})=\left(\frac{\|\mathbf{C}(\boldsymbol{\delta})\|}{\|\mathbf{Q}\|}\right)^{2}, \\
f_{6}(\boldsymbol{\delta})=\left(\frac{\left\|\mathbf{B}^{*}(\boldsymbol{\delta})\right\|}{\|\mathbf{Q}\|}\right)^{2},
\end{gathered}
$$

where $f_{1}(\boldsymbol{\delta})$ represents the elastic requirement on the orthotropy of the laminate having the prescribed shape (imposed by the value of $K^{A^{*}}$ which is related to the sign of $\rho_{0}$ at the end of the first step of the strategy), $f_{2}(\boldsymbol{\delta}), f_{3}(\boldsymbol{\delta})$ and $f_{4}(\boldsymbol{\delta})$ are the requirements related to the prescribed values of the optimal polar parameters resulting from the first-level problem, while $f_{5}(\boldsymbol{\delta})$ and $f_{6}(\boldsymbol{\delta})$ are linked to the quasi-homogeneity condition.

$I\left(f_{i}(\boldsymbol{\delta})\right)$ is a positive semi-definite convex function in the space of laminate polar parameters, since it is defined as a sum of convex functions, see Eqs. (19)-(20). Nevertheless, such a function is highly non-convex in the space of plies orientations because the laminate polar parameters depend upon circular functions of the layers orientation angles. Moreover, the absolute minima of $I\left(f_{i}(\boldsymbol{\delta})\right)$ are known a priori since they are the zeroes of this function. For more details about the nature of the second-level problem see [11, 21]. It is noteworthy that problem (18) must be solved two times, i.e., for each laminate composing the skin and the stiffener.

In order to simplify the problem of retrieving an optimum stack, the search space for problem (18) has been restricted to a particular class of quasi-homogeneous laminates: the quasi-trivial (QT) stacking sequences which constitute exact solutions with respect to the requirements of quasi-homogeneity, i.e., functions $f_{5}(\boldsymbol{\delta})$ and $f_{6}(\boldsymbol{\delta})$ in Eq. (20) are identically null for QT stacks.

QT solutions can be found for laminates with identical plies by acting only on the position of the layers within the stack. Indeed, QT stacks are exact solutions, in terms of quasi-homogeneity condition, regardless to the value of the orientation angle assigned to each layer. In this way orientations represent free parameters which can be optimised to fulfil further elastic requirements, i.e., functions $f_{1}(\boldsymbol{\delta}), f_{2}(\boldsymbol{\delta}), f_{3}(\boldsymbol{\delta})$ and $f_{4}(\boldsymbol{\delta})$. The procedure for searching QT stacks is conceptually simple. Let $n$ be the number of layers and $n_{g} \leq n$ the number of saturated groups. Plies belonging to a given saturated group share the same orientation angle $\theta_{j},\left(j=1, \ldots, n_{g}\right)$. The idea is to look for all the permutations of the position of the plies indexes belongin to each group which meet the quasi-homogeneity condition. More details on this topic can be found in [34].

Suppose now to fix both the number of plies and saturated groups, namely $n$ and $n_{g}$. As discussed in [34], the problem of determining QT stacks for a given couple of $n$ and $n_{g}$ can give rise to a huge number of solutions: the number of QT stacks rapidly increases along with $n$. To this purpose a database of QT stacks has been built for different combinations of $n$ and $n_{g}$.

For the problem at hand, and for each considered case (i.e., skin and stringer laminates), the optimum number of plies $n_{\alpha},(\alpha=S, B)$ constitutes a result of the first-level problem, while the number of saturated groups $n_{g}$ has been fixed a priori. Let be $n_{\text {sol }}$ the number of QT stacks for a particular combination of $n_{\alpha}$ and $n_{g}$. Each solution collected within the database is uniquely defined by means of an identifier ID $_{\text {sol }}$ (i.e., an integer) which varies 
in the range $\left[1, n_{\text {sol }}\right]$. Therefore, $\mathrm{ID}_{\text {sol }}$ represents a further design variable along with the $n_{g}$ orientation angles of the different saturated groups, i.e., $\boldsymbol{\theta} \in \mathbb{R}^{n_{g}}$. The design variables can be thus collected into the following vector,

$$
\boldsymbol{\eta}^{T}=\left\{\mathrm{ID}_{\text {sol }}, \theta_{1}, \ldots, \theta_{n_{g}}\right\},
$$

and problem (18) can be reformulated as

$$
\min _{\boldsymbol{\eta}} \sum_{i=1}^{4} f_{i}(\boldsymbol{\eta}),
$$

$f_{5}(\boldsymbol{\eta})$ and $f_{6}(\boldsymbol{\eta})$ being identically null.

In this background, the solution search for problem (22) is performed by means of the GA BIANCA. In the case of QT stacks the structure of the individual genotype is simple because it is composed of a single chromosome with $n_{g}+1$ genes: the first one codes the variable $\mathrm{ID}_{\text {sol }}$ whilst the remaining genes code the orientation angles of every saturated group which are discrete variables in the range $\left[-89^{\circ}, 90^{\circ}\right]$ with a step length equal to $1^{\circ}$.

\section{Finite element models of the stiffened panel}

In this section two FE models of the stiffened panel RU are discussed: the first one is used in the framework of the first-level problem of the MS2L approach while the second one is utilised for verification purposes.

\subsection{The finite element model for the optimisation procedure}

The FE model of the panel RU used at the first-level of the MS2L strategy is built using the FE commercial code ANSYS ${ }^{\circledR}$. A linear eigenvalue buckling analysis is conducted to determine the value of the first buckling load for each individual, i.e., for each point in the design space, at the current generation.

The need to analyse, within the same generation, different geometrical configurations (RUs with different geometrical and mechanical properties), each one corresponding to an individual, requires the creation of an $a d-h o c$ input file for the $\mathrm{FE}$ code that has to be interfaced with BIANCA. The FE model must be conceived to take into account for a variable geometry, material and mesh. Indeed, for each individual at the current generation, the $\mathrm{FE}$ code has to be able to vary in the correct way the previous quantities, thus a proper parametrisation of the model has to be achieved.

The FE model of the RU is illustrated in Fig. 3. The model has been built by using a combination of eight-nodes shell elements (ANSYS SHELL281 elements) and non-linear multi-point constraints elements (ANSYS MPC184 elements) both with six Degrees Of Freedom (DOFs) per node.

As far as concerns SHELL281 elements, their mechanical behaviour is described by defining directly the homogenised stiffness matrices $\mathbf{A}^{*}, \mathbf{B}^{*}, \mathbf{D}^{*}$ and $\mathbf{H}^{*}$.

The compatibility of the displacement field between skin and stringer is achieved through ANSYS MPC184 elements whose formulation is based upon a classical multi-point constraint element scheme [35]. MPC184 elements are defined between each couple of nodes belonging to contiguous shell elements as depicted in Fig. 3. In particular, MPC184 elements are defined between nodes of the middle plane of the skin (master nodes) and those of the middle plane of the bottom flanges of the stringer (slave nodes). 
Furthermore, MPC184 elements have been utilised to rigidify the end transverse sections of the $\mathrm{RU}$, in order to simulate the presence of ribs (these last having an in-plane stiffness one/two order of magnitude higher than the flexural stiffness of the RU). In particular, two pilot nodes $\mathrm{A}=\{0,0, \hat{z}\}$ and $\mathrm{B}=\{b, 0, \hat{z}\}$ have been defined according to the RU global frame depicted in Fig. 3 ( $\hat{z}$ is the $z$ component of the barycentre of lines belonging to a given transverse section). Then, nodes $\mathrm{A}$ and $\mathrm{B}$ have been connected (through MPC184 elements) to those located on lines of the corresponding transverse section, i.e., lines belonging to the planes $x=0$ and $x=b$, respectively (see Fig. 3). The BCS for nodes $\mathrm{A}$ and $\mathrm{B}$ are

$$
\begin{array}{ll}
\text { node A: } & u_{i}=0, \beta_{i}=0 \\
\text { node B: } & F_{x}=-1 \mathrm{~N}, u_{y}=u_{z}=0, \beta_{i}=0, \\
(i=x, y, z) . &
\end{array}
$$

In Eq. (23) $u_{i}$ and $\beta_{i}$ are nodal displacements and rotations, respectively, whilst $F_{x}$ is the $x$ component of the nodal force.

It is noteworthy that in problem (17) the first-buckling load of the stiffened panel is calculated by considering pertinent $\mathrm{BCs}$ on its RU. This fact implicitly implies the hypothesis of a panel having an "infinite" length along $y$-axis, according to the frame depicted in Fig. 3. To take into account for this aspect, periodic boundary conditions (PBCs) must be considered:

$$
\begin{aligned}
& \left.u_{i}\left(x,-\frac{a}{2}, 0\right)-u_{i}\left(x, \frac{a}{2}, 0\right)=0, \forall x \in\right] 0, b[ \\
& \left.\beta_{i}\left(x,-\frac{a}{2}, 0\right)-\beta_{i}\left(x, \frac{a}{2}, 0\right)=0, \forall x \in\right] 0, b[ \\
& (i=x, y, z) .
\end{aligned}
$$

PBCs of Eq. (24) must be defined for each couple of nodes belonging to the skin lateral edges (i.e., lines located at $y= \pm a / 2$ ) except those placed on the lines at $x=0$ and $x=b$, these last being already connected to the pilot nodes A and B, respectively. PBCs are defined through ANSYS constraint equations (CEs) [35] between homologous nodes of the skin lateral edges

Finally, before starting the optimisation process, a sensitivity study (not reported here for the sake of brevity) on the proposed FE model with respect to the mesh size has been conducted: it was observed that a mesh having 56959 DOFs is sufficient to properly evaluate the first buckling load of the stiffened panel.

\subsection{The enhanced finite element model for the verification phase}

The validity and accuracy of the ANSYS model utilised within the optimisation procedure is verified a-posteriori in this work, by using an advanced higher-order formulation. This refined solutions make use of the Carrera Unified Formulation (CUF), according to which the three-dimensional displacement field $\mathbf{u}(x, y, z)$ can be expressed as a general expansion of the primary unknowns. In the case of one-dimensional theories, one has:

$$
\mathbf{u}(x, y, z)=F_{\tau}(y, z) \mathbf{u}_{\tau}(x), \quad \tau=1,2, \ldots, M
$$


where $F_{\tau}$ are arbitrary functions of the coordinates $y$ and $z$ on the cross-section of the beam structure, $\mathbf{u}_{\tau}$ is the vector of the generalized displacements which lay along the beam axis $x$ and $M$ stands for the number of terms used in the high-order expansion. To be remarked that in Eq. (25) (as well as in the rest of the equations of this subsection) Einstein summation convention on repeated indices is tacitly assumed.

The choice of $F_{\tau}$ determines the class of the 1D CUF model that is required and subsequently to be adopted. For example, if Lagrange polynomials are used as $F_{\tau}$, Layer-Wise (LW) theories for composite structures can be easily implemented, see [36]. Unlike classical models for laminates which are available in commercial software tools, the unknowns of the problem (and, thus, the number of DOFs) are layer-dependent in the case of LW models. In this manner, it is possible to satisfy the continuity of the transverse stresses and the zig-zag behaviour of the displacements along the thickness of the composite structure, in accordance with the equilibrium and compatibility equations of elasticity.

One of the most important advantages of CUF is that it allows to write the governing equations and the related finite element arrays of low-order to high-fidelity LW models in an unified manner. Generally speaking, CUF can be used to generate finite elements whose formal mathematical expressions are independent of the theory kinematics. For example, in this work, the critical buckling loads are calculated by linearising the geometric nonlinear governing equations and evaluating the loads that make the linearised tangent stiffness matrix singular; i.e. $\left|\mathbf{K}_{T}\right| \approx\left|\mathbf{K}+\mathbf{K}_{\sigma}\right|=0$, where $\mathbf{K}$ is the linear stiffness matrix and $\mathbf{K}_{\sigma}$ is the geometric stiffness matrix.

The linear stiffness matrix can be evaluated from the virtual variation of the internal work, which holds

$$
\delta L_{i n t}=\int_{l} \int_{\Omega} \delta \boldsymbol{\epsilon}^{T} \boldsymbol{\sigma} d V
$$

where $\boldsymbol{\epsilon}$ and $\boldsymbol{\sigma}$ are the strain and stress vectors (Voigt's notation), $\Omega$ is the cross-section of the beam structure and $l$ is the beam length. By substituting the constitutive and linear geometrical relations as well as CUF (Eq. (25)) and a classical finite element approximation along the beam axis $x$, such that $\mathbf{u}_{\tau}(x)=N_{i}(x) \mathbf{u}_{\tau i}$, the virtual variation of the strain energy reads:

$$
\delta L_{i n t}=\delta \mathbf{u}_{\tau i}^{T} \mathbf{K}^{i j \tau s} \mathbf{u}_{s j}
$$

where $\mathbf{u}_{\tau i}$ is the vector of the finite element unknowns and $i$ represents summation on the nodes of the beam element. $\mathbf{K}^{i j \tau s}$ represents the $3 \times 3$ fundamental nucleus of the stiffness matrix, which can be expanded according to $(i, j)$ and $(\tau, s)$ to obtain the finite element array of the generic beam theory [37]. Similarly, the gometric stiffness matrix $\mathbf{K}_{\sigma}$ can be expressed in terms of fundamental nucleus by evaluating the linearisation of the virtual variation of the strain energy and, subsequently, by linearising the nonlinear geometric relations [38]. This matrix, in fact, represents the contribution of the pre-stress on the stiffness of the system. It is important to underline that, in this work, as accurate LW models of the reinforced composite panels are implemented, the full three-dimensional stress field is taken into account for evaluating the geometric stiffness matrix $\mathbf{K}_{\sigma}$. This is not true in the case of the ANSYS model employed in the optimisation procedure, which makes use of standard shell elements based on FSDT assumptions. 


\section{Numerical results}

Before starting the multi-scale optimisation process a reference structure must be defined in order to establish reference values for the RU mass as well as for the first buckling load of the stiffened panel: both material and geometrical properties of the reference solution are reported in Tables 1 and 3, respectively. The reference solution is subject to the same set of BCs, i.e., Eqs. (23) and (24), as those applied on the RU of the panel that will be optimised. One can notice that the reference structure has a laminated skin composed of 28 plies and disposed according to a symmetric, balanced stack (therefore the resulting laminate is uncoupled and orthotropic in membrane, but not in bending), whilst the stringer laminate is made of 32 plies with a symmetric quasi-isotropic stack (the laminate is uncoupled and the membrane stiffness matrix is isotropic, but the bending one is totally anisotropic). This reference solution corresponds to a classical configuration utilised in the aeronautical field: its mass and its stiffness properties (in terms of buckling load) still represent a "good" compromise between weight and stiffness requirements.

Regarding the setting of the genetic parameters for the GA BIANCA utilised to perform the solution search for both first and second-level problems they are listed in Table 4. Moreover, concerning the constraint-handling technique for the first-level problem the Automatic Dynamic Penalisation (ADP) method has been considered, see [29]. For more details on the numerical techniques developed within the new version of BIANCA and the meaning of the values of the different parameters tuning the GA the reader is addressed to $[20]$.

\subsection{Optimum configurations of the panel}

The optimum values of both geometric and mechanical design variables (dimensionless variables) resulting from the first-level of the optimisation strategy are listed in Table 5. When comparing the optimum solution of the first-level problem with the reference configuration, one can notice the number of plies reduces from 28 to 20 for the skin laminate and from 32 to 28 for the stringer. Moreover, both laminates are quasi-homogeneous and fully orthtropic (both membrane and bending stiffness matrices) with an ordinary orthotropy shape (parameter $K^{A^{*}}=0$ because the anisotropic polar modulus $R_{0 K}^{A *}$ is positive for both cases, see [11]). However, skin laminate gets a lower value of polar parameter $R_{1}^{A *}$ (an order of magnitude lower than the corresponding value of $R_{0 K}^{A *}$ ) which means that this solutions tends to exhibit a square symmetric behaviour (for both membrane and bending stiffness matrices), as illustrated in the polar diagrams of Fig. 4. For a deeper insight on these aspects the interested reader is addressed to [11, 21].

Table 6 reports the first two best stacking sequences, for both skin and stringer, which represents just as many solution for problem (22). As stated in Section 4 the secondlevel problem is solved in the space of QT stacks. In this background, after fixing the number of plies $n$ and the number of saturated groups $n_{g}$ the design variables are the identifier of the QT solution as well as the orientation angle of each saturated group, see Eq. (21). Because problem (22) is highly non-convex in the space of the orientation angles of saturated groups, it is possible to find several solutions (theoretically an infinite number) meeting the optimum value of the laminate polar parameters provided by the first-level problem.

For the problem at hand, the number of plies for both skin and stringer laminates, ( $n_{S}$ and $n_{B}$, respectively) is a direct result of the first level problem, while the number of saturated group has been set equal to

- three for stacks S1 and B1, 
- four for stack S2,

- five for stack B2.

As it can be easily inferred from the results listed in Table 7 , by combining the previous stacks it is possible to get four different optimum configurations of the stiffened panel. Indeed, these optimum panels really represent equivalent solutions. Since they share the same macroscopic geometrical parameters they have the same mass, i.e., $M=0.814 \mathrm{Kg}$ which represents a significant reduction $(-11.5 \%)$ when compared to the reference configuration. Furthermore, these optimal configurations differ only in terms of the optimum stack composing skin and stiffener laminates but they show almost the same buckling response: the percentage increment of the first buckling load (with respect to the reference value $\lambda_{\text {ref }}$ ) ranges from $9 \%$ to $9.5 \%$, see Table 7 .

Therefore, each optimum configuration is simultaneously lighter and stiffer than the reference one and this result has been achieved only by abandoning the usual engineering rules and hypotheses related to the nature of the stacking sequence of the laminates composing the panel.

Fig. 4 shows the deformed shape related to the first buckling mode as well as the first component of the normalised stiffness matrices of the laminate, i.e., $\mathbf{A}^{*}, \mathbf{B}^{*}$ and $\mathbf{D}^{*}$ for both skin and stringer for the configuration S1-B1: the solid line refers to the membrane stiffness matrix, the dashed one to the bending stiffness matrix, while the dash-dotted one is linked to the membrane/bending coupling stiffness matrix. It can be noticed that the laminate is uncoupled as the dash-dotted curve disappears, homogeneous as the solid and dashed curves are coincident and orthotropic because there are two orthogonal axes of symmetry in the plane. In addition, for both laminates the main orthotropy axis is oriented at $\Phi_{1}^{A^{*}}=0^{\circ}$ according to the hypothesis of the first-level problem. The same considerations can be repeated also for the rest of the optimum solutions.

\subsection{Verification of the optimum configurations}

A one-dimensional, high-order model based on CUF is used for validating the reference and optimised RU analyses. The present CUF model employs a LW refined kinematics for the accurate description of the pre-stress state of the RU subjected to compression and, thus, for enhanced evaluation of buckling loads. The CUF-LW models of the reference and optimised RU panels have 372588 and 333792 DOFs, respectively. As in the case of the ANSYS model, PBCs are imposed by using the direct penalty approach. However, it is important to underline that, because the employed LW CUF models have only pure translational displacements as unknowns, only the first line of Eq. (24) is enforced.

The first buckling mode of the optimum configuration S1-B1 is shown in Fig. 5. That of the reference configuration as well as those associated to the other optimum solutions are equivalent, thus they are not depicted for the sake of brevity. For completeness reasons, however, the through-the-thickness stress distributions (see Fig. 6) according to CUF and ANSYS are given in Figs. 7 and 8. These figures show the distributions of axial, $\sigma_{x x}$, transverse shear, $\sigma_{x z}$, and transverse normal, $\sigma_{z z}$, stress components. It should be underlined that the adopted ANSYS model provides a good distribution of axial stresses. In contrast, and according to CUF reference solutions, the ANSYS FE model is not able to take into account shear and transverse normal stresses and this would directly affect the accuracy of the buckling calculation.

Table 8 summarises the first critical buckling loads given by CUF high-order beam models and they are compared to those resulting from ANSYS model. The differences between the results of the ANSYS FE model and the refined CUF solution for the optimum 
panels range from $7.4 \%$ to $7.9 \%$, while for the reference configuration the percentage difference is significant (up to 14\%). This higher discrepancy is probably related to the anisotropic bending behaviour of the reference solution. These differences are reasonable and are related to the 3D stress distributions within each constitutive layer and the different order of accuracy characterising the CUF LW beam model. Of course, this stress field strongly affects the geometric stiffness matrix and cannot be acquired by ANSYS shell elements which are based on the FSDT hypotheses.

It is noteworthy that, according to CUF numerical results, the gain in terms of stiffness is even higher than that foreseen by ANSYS, ranging from $15.2 \%$ for solution B1-S1 to $15.8 \%$ for solution B2-S2, as summarised in Table 9.

\section{Conclusions}

The design strategy presented in this paper is a numerical optimisation procedure characterised by several features that make it an innovative, effective and general method for the multi-scale design of composite structures. In the present work this strategy has been applied to the multi-scale optimisation of the repetitive unit of a composite stiffened panel.

On the one hand, the design process is not submitted to restrictions: any parameter characterising the structure (at each relevant scale) is an optimisation variable. This allows searching for a true global minimum without making simplifying hypotheses on the nature of the laminate stacking sequence. On the other hand, the multi-scale design problem has been split into two optimisation sub-problems which are solved subsequently within the same numerical procedure.

The first-level problem focuses on the macroscopic scale of the panel: each laminate composing the structure is considered as an equivalent homogeneous anisotropic plate (for both skin and stringer) and its macroscopic mechanical response is described in terms of polar parameters. Furthermore, also geometric design variables describing the topology of both skin and stiffener are involved at this level. At this stage, the mechanical properties of the multilayer plates are represented by means of the polar formalism, a mathematical representation based on tensor invariants which is characterised by several advantages. The main features of the polar method are the possibility to represent in an explicit and straightforward way the elastic symmetries of the laminate stiffness matrices and to eliminate from the optimisation procedure redundant mechanical properties.

The second level of the procedure is devoted to the laminate mesoscopic scale: the goal is to find at least one optimum stack (for both stringer and skin) meeting on the one hand the elastic requirements imposed to the laminate (quasi-homogeneity and orthotropy) during the first-level problem and on the other hand the optimum value of the laminate polar parameters resulting from the first step.

The utilisation of an evolutionary strategy, together with the fact that the problem is stated in the most general sense, allows finding some non-conventional configurations more efficient than the standard ones. In fact, the considered numerical example proves that, when standard rules for tailoring laminate stacks are abandoned and all the parameters characterising the structure are included within the design process, a significant weight saving can be obtained: up to $11.5 \%$ with respect to the reference structure with enhanced mechanical properties in terms of first buckling load (the percentage increment ranges from $9 \%$ to $9.5 \%$ depending on the considered optimum solution).

In a second time, both reference and optimum configurations of the stiffened panel have been analysed by means of a high-order layer-wise FE model developed in the framework 
of CUF. This analysis reveals that the buckling load provided by the ANSYS FE model (which is built by using shell elements based on FSDT) is overestimated and that the percentage difference ranges from $7.4 \div 7.9 \%$ for optimum solutions to $14 \%$ for the reference configuration. This discrepancy is related to the calculation of the 3D stress field in each layer which strongly affects the geometric stiffness matrix used to evaluate the first buckling load of the panel.

Nevertheless, despite these discrepancies, classical shell elements based on FSDT can be reliably employed in the framework of the MS2L optimisation strategy because they allow finding true optimum solutions without using "expensive" models, in terms of both number of DOFs and computational cost. Moreover, according to CUF results, the optimum configurations are really efficient when compared to the reference one: the weight saving is always the same, but the gain in terms of stiffness is even higher than that foreseen by ANSYS, ranging from $15.2 \%$ to $15.8 \%$ depending on the optimum solution.

These results unquestionably prove the effectiveness and the robustness of the optimisation approach proposed in this work and provide confidence for further research in this direction. As an example, future works may focus on coupling the present MS2L optimisation strategy with high-order models based on CUF. These considerations remain still valid if further requirements (e.g., strength, fatigue, delamination, etc.) have to be included into the design problem formulation. All of these aspects can be easily integrated within the MS2L optimisation strategy without altering its overall architecture and they do not represent a limitation to the proposed strategy, on the contrary they could be an interesting challenge for future researches on real-world engineering applications.

\section{Acknowledgements}

G. A. Fiordilino is grateful to the Nouvelle-Aquitaine region for its contribution to this paper through the SMARTCOMPOSITE project. A. Pagani also acknowledges financial support from the Compagnia di San Paolo and Politecnico di Torino trough the project ADAMUS.

\section{References}

[1] S. Nagendra, D. Jestin, Z. Gürdal, R. Haftka, L. Watson, Improved genetic algorithm for the design of stiffened composite panels, Computers \& Structures 58 (3) (1996) $543-555$.

[2] C. Bisagni, L. Lanzi, Post-buckling optimisation of composite stiffened panels using neural networks, Composite Structures 58 (2002) 237-247.

[3] L. Lanzi, V. Giavotto, Post-buckling optimization of composite stiffened panels: Computations and experiments, Composite Structures 73 (2006) 208-220.

[4] E. Barkanov, O. Ozolins, E. Eglitis, F. Almeida, M. Bowering, G. Watson, Optimal design of composite lateral wing upper covers. Part I: linear buckling analysis, Aerospace Science and Technology 38 (2014) 1-8.

[5] D. Liu, V. Toropov, M. Zhou, D. Barton, O. Querin, Collection of Technical Papers AIAA/ASME/ASCE/AHS/ASC Structures, Structural Dynamics and Materials Conference, 2010, Ch. Optimization of Blended Composite Wing Panels Using Smeared Stiffness Technique and Lamination Parameters. 
[6] C. López, O. Bacarreza, A. Baldomir, S. Hernandez, M. Aliabadi, Reliability-based design optimization of composite stiffened panels in post-buckling regime 55 (3) (2016) $1121-1141$.

[7] M. Lillico, R. Butler, G. Hunt, A. Watson, D. Kennedy, F. Williams, Optimum design and testing of a post-buckled stiffened panel., American Institute of Aeronautics and Astronautics, AIAA-2000-1659 (2000) 1-10.

[8] R. Butler, F. Williams, Optimum design using viconopt, a buckling and strength constraint program for prismatic assemblies of anisotropic plates, Computers \& Structures 43 (1992) 699-708.

[9] J. Wiggenraad, P. Arendsen, J. da Silva Pereira, Design optimisation of stiffened composite panels with buckling and damage tolerance constraints, American Institute of Aeronautics and Astronautics Journal (1998) 420-430.

[10] P. Kaletta, K. Wolf, Optimisation of composite aircraft panels using evolutionary computation methods, Proceedings of ICAS 2000 Congress, Harrogate, UK, 27 August-1 September (2000) 441.1-10.

[11] M. Montemurro, An extension of the polar method to the first-order shear deformation theory of laminates, Composite Structures 127 (2015) 328-339.

[12] S. Nagendra, D. Jestin, Z. Gürdal, R. Hatfka, L. Watson, Improved genetic algorithm for the design of stiffened composite panels, Computers and Structures 58 (3) (1996) $543-555$.

[13] R. Le Riche, R. T. Hatfka, Improved genetic algorithm for minimum thickness composite laminate design, Composites Engineering 5 (2) (1995) 143-161.

[14] A. Todoroki, R. T. Hatfka, Stacking sequence optimization by a genetic algorithm with a new recessive gene like repair strategy, Composites Part B 29 (1998) 277-285.

[15] B. Liu, R. T. Hatfka, P. Trompette, Maximization of buckling loads of composite panels using flexural lamination parameters, Structural and Multidisciplinary Optimization 26 (2004) 28-36.

[16] A. Catapano, M. Montemurro, A multi-scale approach for the optimum design of sandwich plates with honeycomb core. Part I: homogenisation of core properties, Composite Structures 118 (2014) 664-676.

[17] A. Catapano, M. Montemurro, A multi-scale approach for the optimum design of sandwich plates with honeycomb core. Part II: the optimisation strategy, Composite Structures 118 (2014) 677-690.

[18] M. Montemurro, A. Catapano, D. Doroszewski, A multi-scale approach for the simultaneous shape and material optimisation of sandwich panels with cellular core, Composites Part B 91 (2016) 458-472.

[19] G. Verchery, Les invariants des tenseurs d'ordre 4 du type de l'élasticité, Proc. of colloque Euromech 115, VIllard-de-Lans, (France), 1979.

[20] M. Montemurro, Optimal design of advanced engineering modular systems through a new genetic approach, Ph.D. thesis, UPMC, Paris VI, France, http://tel.archivesouvertes.fr/tel-00955533 (2012). 
[21] M. Montemurro, Corrigendum to "an extension of the polar method to the first-order shear deformation theory of laminates" [compos. struct. 127 (2015) 328-339], Composite Structures 131 (2015) 1143-1144.

[22] M. Montemurro, The polar analysis of the third-order shear deformation theory of laminates, Composite Structures 131 (2015) 775-789.

[23] M. Montemurro, A. Catapano, Variational analysis and aerospace engineering: mathematical challenges for the aerospace of the future, 1st Edition, Vol. 116 of Springer Optimization and Its Applications, Springer International Publishing, 2016, Ch. A new paradigm for the optimum design of variable angle tow laminates, DOI: $10.1007 / 987$ 3-319-45679-9.

[24] M. Montemurro, A. Catapano, On the effective integration of manufacturability constraints within the multi-scale methodology for designing variable angle-tow laminates, Composite Structures 161 (2017) 145-159.

[25] D. P. Raymer, Aircraft Design: A Conceptual Approach, American Institute of Aeronautics \& Astronautics, 2013.

[26] J. N. Reddy, Mechanics of composite laminated plates and shells: theory and analysis, Boca Raton, FL: CRC Press, 2003.

[27] P. Vannucci, Plane anisotropy by the polar method, Meccanica 40 (2005) 437-454.

[28] P. Vannucci, A note on the elastic and geometric bounds for composite laminates, Journal of Elasticity 112 (2013) 199-215.

[29] M. Montemurro, A. Vincenti, P. Vannucci, The automatic dynamic penalisation method (ADP) for handling constraints with genetic algorithms, Computer Methods in Applied Mechanics and Engineering 256 (2013) 70-87.

[30] M. Montemurro, A. Vincenti, Y. Koutsawa, P. Vannucci, A two-level procedure for the global optimization of the damping behavior of composite laminated plates with elastomer patches, Journal of Vibration and Control 21 (9) (2015) 1778-1800.

[31] M. Montemurro, Y. Koutsawa, S. Belouettar, A. Vincenti, P. Vannucci, Design of damping properties of hybrid laminates through a global optimisation strategy, Composite Structures 94 (2012) 3309-3320.

[32] M. Montemurro, H. Nasser, Y. Koutsawa, S. Belouettar, A. Vincenti, P. Vannucci, Identification of electromechanical properties of piezoelectric structures through evolutionary optimisation techniques, International Journal of Solids and Structures 49 (13) (2012) 1884-1892.

[33] A. Catapano, Stiffness and strength optimisation of the anisotropy distribution for laminated structures, Ph.D. thesis, UPMC, Paris VI, France, http://tel.archivesouvertes.fr/tel-00952372 (2013).

[34] P. Vannucci, G. Verchery, A special class of uncoupled and quasi-homogeneous laminates, Composites Science and Technology 61 (2001) 1465-1473.

[35] ANSYS, Inc., 275 Technology Drive, Canonsburg, PA 15317, ANSYS Mechanical APDL Modeling and Meshing Guide (2012). 
[36] E. Carrera, M. Petrolo, Refined one-dimensional formulations for laminated structure analysis, AIAA Journal 50 (1) (2012) 176-189. doi:10.2514/1. J051219.

[37] E. Carrera, M. Cinefra, M. Petrolo, E. Zappino, Finite Element Analysis of Structures through Unified Formulation, John Wiley \& Sons, Chichester, West Sussex, UK, 2014.

[38] A. Pagani, E. Carrera, Unified formulation of geometrically nonlinear refined beam theories, Mechanics of Advanced Materials and Structures 25 (1) (2018) 15-31. 


\section{Tables}

\begin{tabular}{ll|ll|ll}
\hline \multicolumn{2}{c}{ Technical constants } & \multicolumn{2}{c}{ Polar parameters of $\mathbf{Q}^{a}$} & \multicolumn{2}{c}{ Polar parameters of $\hat{\mathbf{Q}}^{b}$} \\
\hline$E_{1}[\mathrm{MPa}]$ & 161000.0 & $T_{0}[\mathrm{MPa}]$ & 23793.3868 & $T[\mathrm{MPa}]$ & 5095.4545 \\
$E_{2}[\mathrm{MPa}]$ & 9000.0 & $T_{1}[\mathrm{MPa}]$ & 21917.8249 & $R[\mathrm{MPa}]$ & 1004.5454 \\
$G_{12}[\mathrm{MPa}]$ & 6100.0 & $R_{0}[\mathrm{MPa}]$ & 17693.3868 & $\Phi[\mathrm{deg}]$ & 90.0 \\
$\nu_{12}$ & 0.26 & $R_{1}[\mathrm{MPa}]$ & 19072.0711 & & \\
$\nu_{23}$ & 0.10 & $\Phi_{0}[\mathrm{deg}]$ & 0.0 & & \\
& & $\Phi_{1}[\mathrm{deg}]$ & 0.0 & &
\end{tabular}

Density and thickness

$\rho\left[\mathrm{Kg} / \mathrm{mm}^{3}\right] \quad 1.58 \times 10^{-6}$

$t_{\text {ply }}[\mathrm{mm}] \quad 0.125$

${ }^{a}$ In-plane reduced stiffness matrix of the ply.

${ }^{b}$ Out-of-plane shear stiffness matrix of the ply.

Table 1: Material properties of the carbon-epoxy ply taken from [11, 21, 22].

\begin{tabular}{lllll}
\hline Design variable & Type & Lower bound & Upper bound & Discretisation step \\
\hline$\rho_{0 S}$ & continuous & -1.0 & 1.0 & - \\
$\rho_{1 S}$ & continuous & 0 & 1.0 & - \\
$\rho_{0 B}$ & continuous & -1.0 & 1.0 & - \\
$\rho_{1 B}$ & continuous & 0 & 1.0 & - \\
$c_{1}$ & discrete & 0.1 & 0.45 & 0.001 \\
$c_{2}$ & discrete & 1.00 & 3.00 & 0.01 \\
$c_{3}$ & discrete & 1.00 & 3.00 & 0.01 \\
$n_{S}$ & integer & 20 & 32 & 1 \\
$n_{B}$ & integer & 20 & 32 & 1 \\
\hline
\end{tabular}

Table 2: Design space of the first-level problem.

\begin{tabular}{lll}
\hline$a[\mathrm{~mm}]$ & 150.00 & \\
$b[\mathrm{~mm}]$ & 600.00 & \\
$a_{2}[\mathrm{~mm}]$ & 15.00 & \\
$a_{3}[\mathrm{~mm}]$ & 21.50 & \\
$h[\mathrm{~mm}]$ & 30.00 & \\
$M_{\text {ref }}[\mathrm{Kg}]$ & 0.92 & \\
$\lambda_{\text {ref }}[\mathrm{N}]$ & 445074 & \\
\hline Stacking sequence & Part & N. of plies \\
\hline$\left[\left(45 /-45 / 90_{2}\right)_{2} /(45 /-45)_{3}\right]_{s}$ & skin $(\mathrm{S})$ & 28 \\
{$\left[45_{2} / 0_{2} /-45_{2} / 90_{4} /-45_{2} / 0_{2} / 45_{2}\right]_{s}$} & stringer $(\mathrm{B})$ & 32 \\
\hline
\end{tabular}

Table 3: Reference solution for the stiffened panel design problem. 


\begin{tabular}{l|l|l}
\hline \multicolumn{3}{c}{ Genetic parameters } \\
\hline & $1^{\text {st }}$ level problem & $2^{\text {nd }}$ level problem \\
\hline N. of populations & 1 & 1 \\
N. of individuals & 200 & 500 \\
N. of generations & 150 & 500 \\
Crossover probability & 0.85 & 0.85 \\
Mutation probability & 0.005 & 0.002 \\
Selection operator & roulette-wheel & roulette-wheel \\
Elitism operator & active & active \\
\hline
\end{tabular}

Table 4: Genetic parameters of the GA BIANCA for first and second-level problems.

\begin{tabular}{|c|c|c|c|c|}
\hline \multicolumn{5}{|c|}{ Geometric parameters } \\
\hline$a_{2}[\mathrm{~mm}]$ & $a_{3}[\mathrm{~mm}]$ & $h[\mathrm{~mm}]$ & $n_{\mathrm{S}}$ & $n_{\mathrm{B}}$ \\
\hline 21.300 & 29.607 & 31.950 & 20 & 28 \\
\hline \multicolumn{5}{|c|}{ Polar parameters } \\
\hline & & & $R_{0 K}^{A *}[\mathrm{MPa}]$ & $R_{1}^{A *}[\mathrm{MPa}]$ \\
\hline & Skin $(\mathrm{S})$ & & 3511.00 & 242.36 \\
\hline & tringer $(\mathrm{B})$ & & 9391.51 & 12080.84 \\
\hline
\end{tabular}

Table 5: Numerical results of the first-level optimisation problem.

\begin{tabular}{|c|c|c|}
\hline ID & Best stacking sequence & N. of plies \\
\hline \multicolumn{3}{|c|}{ Skin (S) } \\
\hline S1 & {$[-63 / 0 / 63 / 0 / 63 /-63 / 0 / 0 / 63 /-63 / 63 /-63 / 0 / 0 / 63 /-63 / 0 /-63 / 0 / 63]$} & 20 \\
\hline $\mathrm{S} 2$ & {$[43 / 90 / 0 / 0 /-43 / 90 /-43 / 90 / 0 /-43 / 43 / 90 / 0 / 43 / 0 / 43 / 90 / 90 / 0 /-43]$} & 20 \\
\hline \multicolumn{3}{|c|}{ Stringer $(\mathrm{B})$} \\
\hline B1 & {$[1 / 61 / 1 / 1 / 1 /-51 / 1 / 1 /-51 / 1 / 1 / 1 / 61 / 1 / 1 /-51 / 1 / 1 / 1 / 61 / 1 / 1 / 61 / 1 / 1 / 1 /-51 / 1]$} & 28 \\
\hline B2 & {$[0 / 59 /-1 /-54 / 2 / 0 / 2 / 2 / 2 / 0 /-54 /-1 / 59 / 2 / 0 / 0 /-54 /-1 / 0 / 59 / 0 / 2 / 59 / 2 /-1 /-54 / 2 / 0]$} & 28 \\
\hline
\end{tabular}

Table 6: Numerical results of the second-level problem (first two optimum stacks for both skin and stringer).

\begin{tabular}{c|c|c|c|c|c}
\hline \multicolumn{6}{c}{ Panel configurations } \\
\hline & REF & S1-B1 & S1-B2 & S2-B1 & S2-B2 \\
\hline$M[\mathrm{Kg}]$ & 0.920 & & $0.814(-11.5 \%)$ & \\
\hline$\lambda[\mathrm{N}]$ & 445074 & $483951(9 \%)$ & $483838(9 \%)$ & $487493(9.5 \%)$ & $487386(9.5 \%)$ \\
\hline
\end{tabular}

Table 7: Properties of the optimum solution (in terms of mass and buckling load) for different skin-stringer configurations; for each property the percentage difference between the optimum configuration and the reference one is indicated in parentheses. 


\begin{tabular}{c|c|c|c|c|c}
\hline \multicolumn{7}{c}{ Panel configurations } \\
\hline$\lambda[\mathrm{N}]$ & REF & S1-B1 & S1-B2 & S2-B1 & S2-B2 \\
\hline CUF & 390870 & 450323 & 450430 & 451843 & 452615 \\
\hline ANSYS & $445074(14 \%)$ & $483951(7.5 \%)$ & $483838(7.4 \%)$ & $487493(7.9 \%)$ & $487386(7.7 \%)$ \\
\hline
\end{tabular}

Table 8: Comparison of the buckling load $\lambda$ calculation between ANSYS FE model and high-order beam CUF model for both reference and optimum solutions; the percentage difference between ANSYS and CUF models is indicated in parentheses.

\begin{tabular}{c|c|c|c|c|c}
\hline \multicolumn{6}{c}{ Panel configurations } \\
\hline & REF & S1-B1 & S1-B2 & S2-B1 & S2-B2 \\
\hline$\lambda[\mathrm{N}]$ & 390870 & $450323(15.2 \%)$ & $450430(15.2 \%)$ & $451843(15.6 \%)$ & $452615(15.8 \%)$ \\
\hline
\end{tabular}

Table 9: Comparison of the buckling load provided by the high-order beam CUF model for both reference and optimum solutions; the percentage difference between each optimum configuration and the reference one is indicated in parentheses. 


\section{Figures}

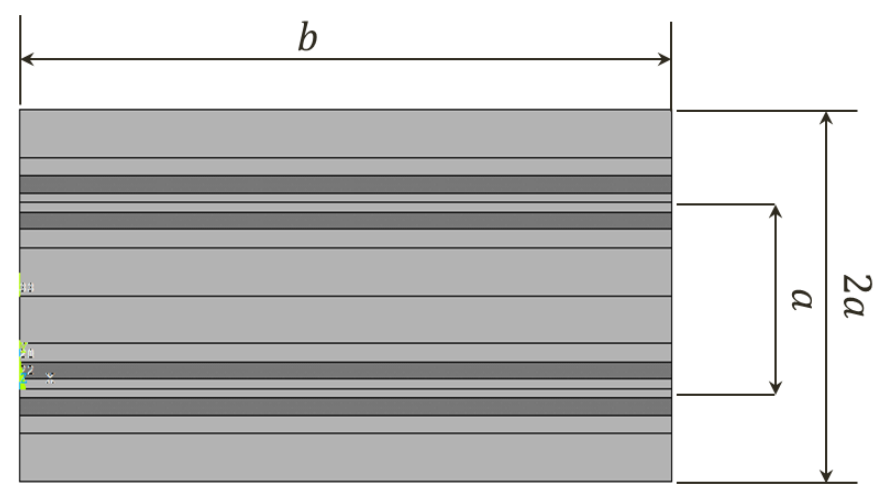

$a_{2} / 2$

(a)



(b)

Figure 1: (a) Geometry and overall size of the stiffened panel (only two repetitive units are here represented for sake of simplicity) and (b) geometric parameters of the repetitive unit. 


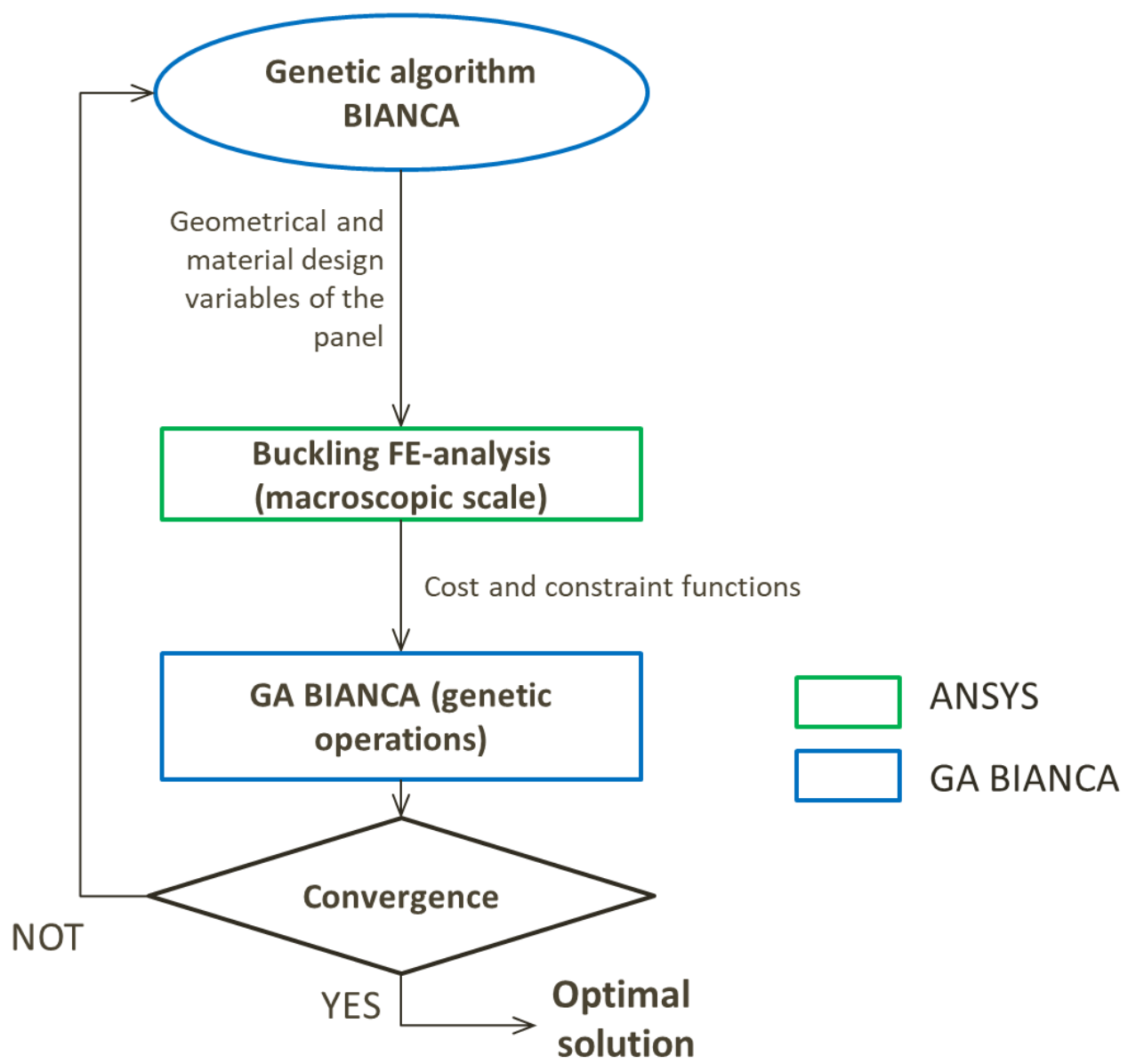

Figure 2: Logical flow of the numerical procedure for the solution search of the first-level problem. 

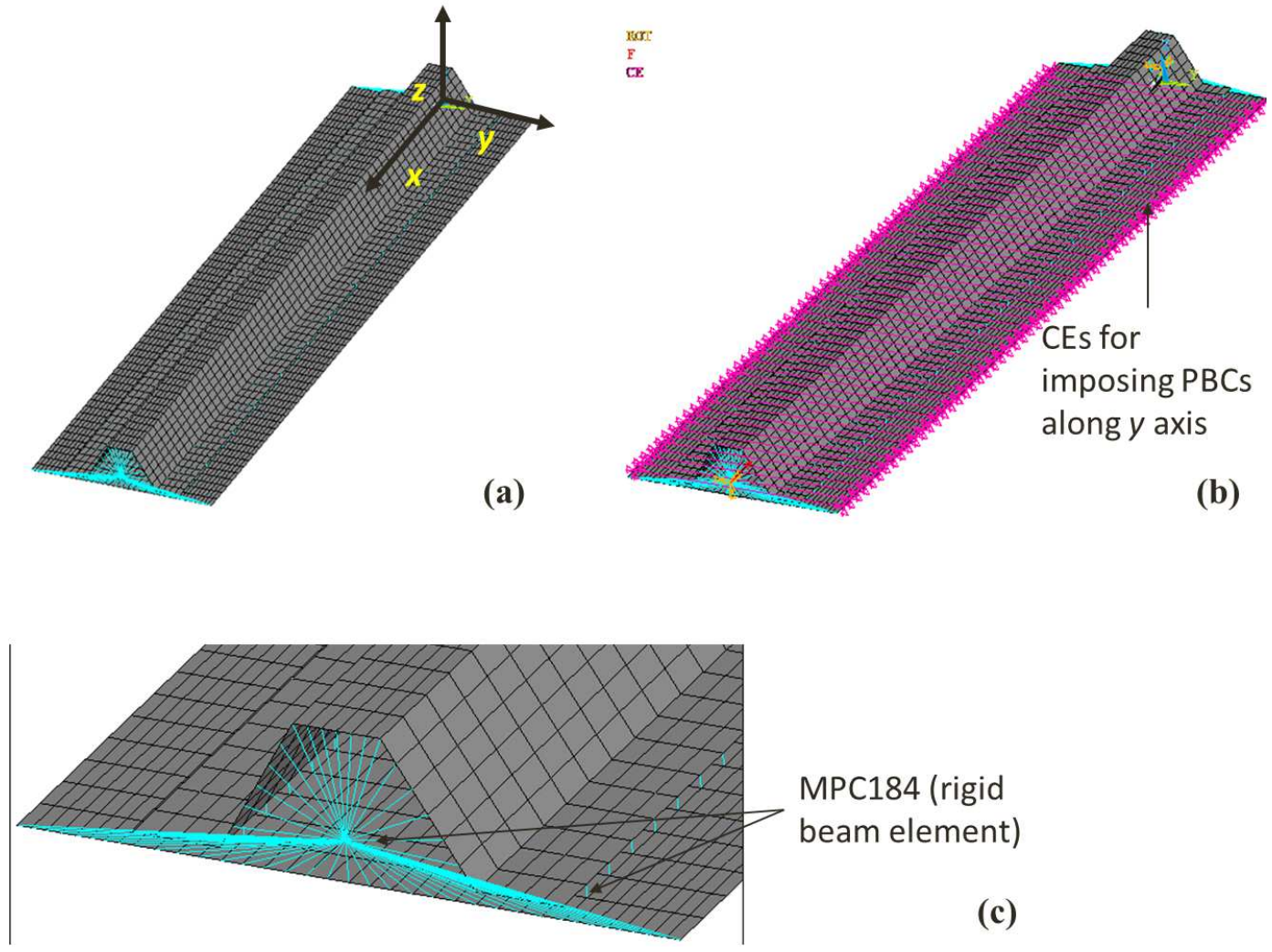

Figure 3: (a) FE model of the repetitive unit and related reference frame, (b) details of CEs for PBCs along $y$-axis and (c) details of MPC184 elements. 

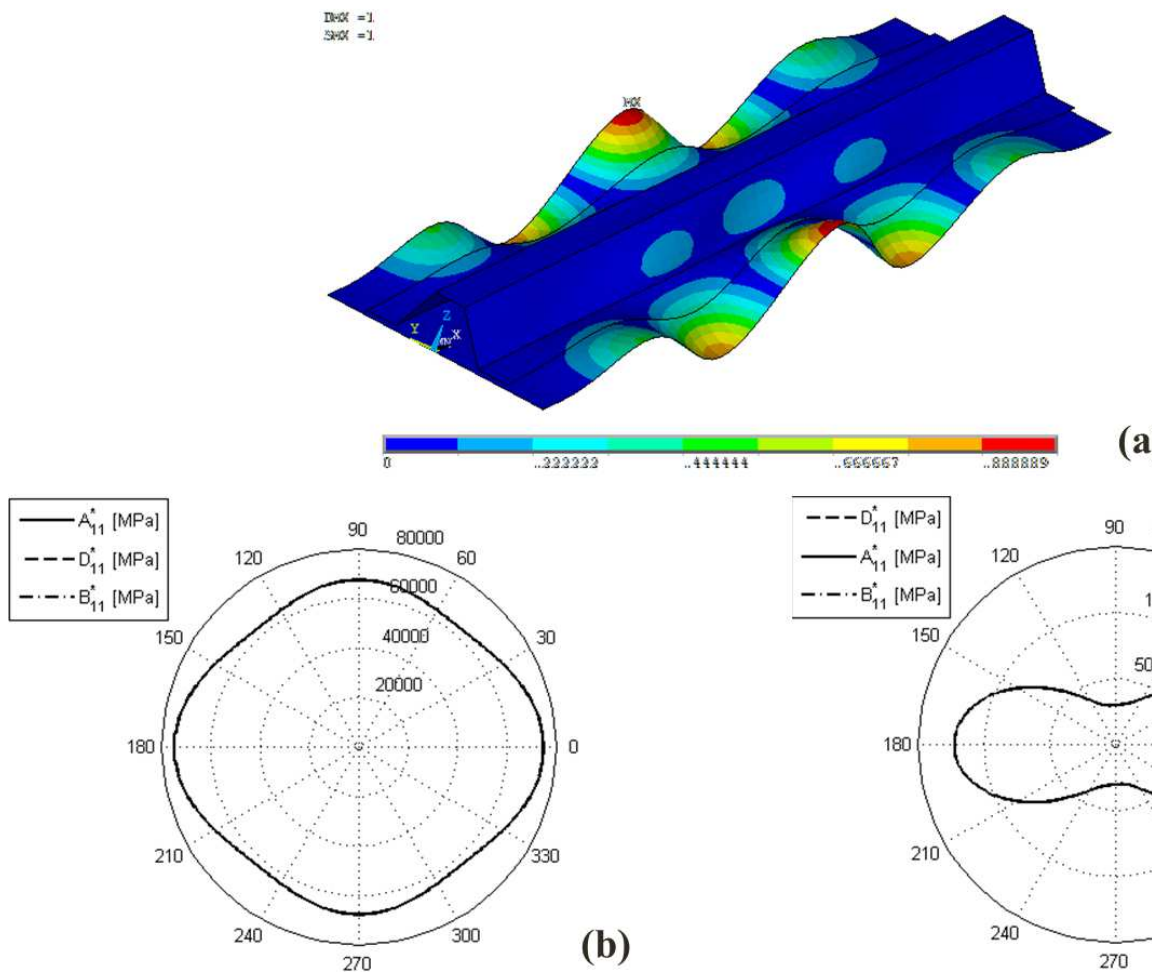

(a)

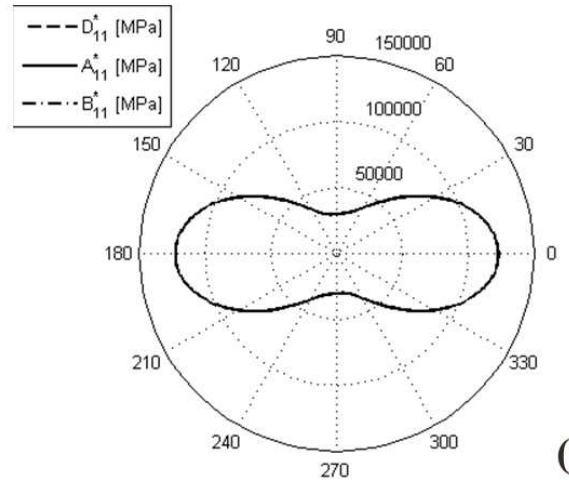

(c)

Figure 4: Numerical properties of the optimum panel S1-B1. (a) Deformed shape of the first buckling mode (normalized displacement) and polar diagram of the first component of the homogenized laminate in-plane stiffness matrices $[\mathrm{MPa}]$ for (b) skin and (c) stringer.

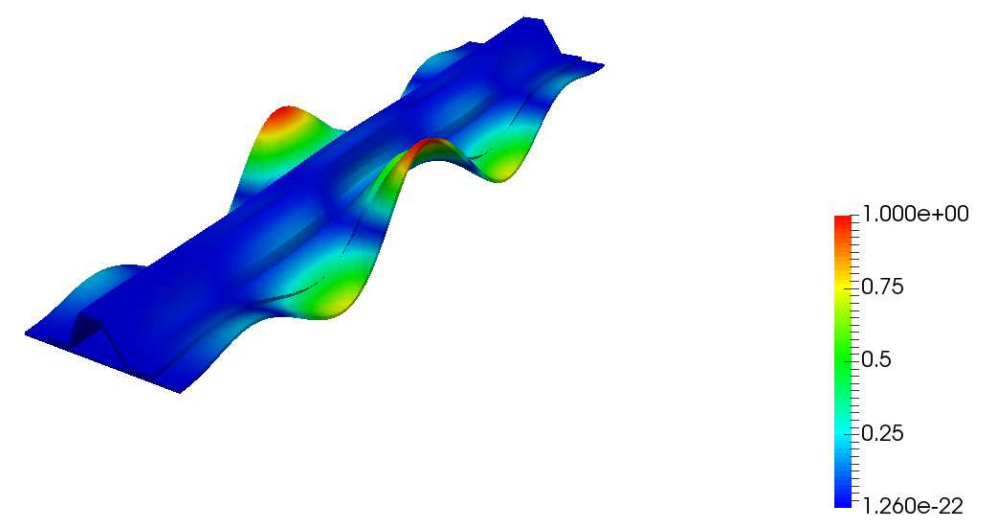

Figure 5: First buckling mode of optimum panel S1-B1 according to higher-order CUF model. 


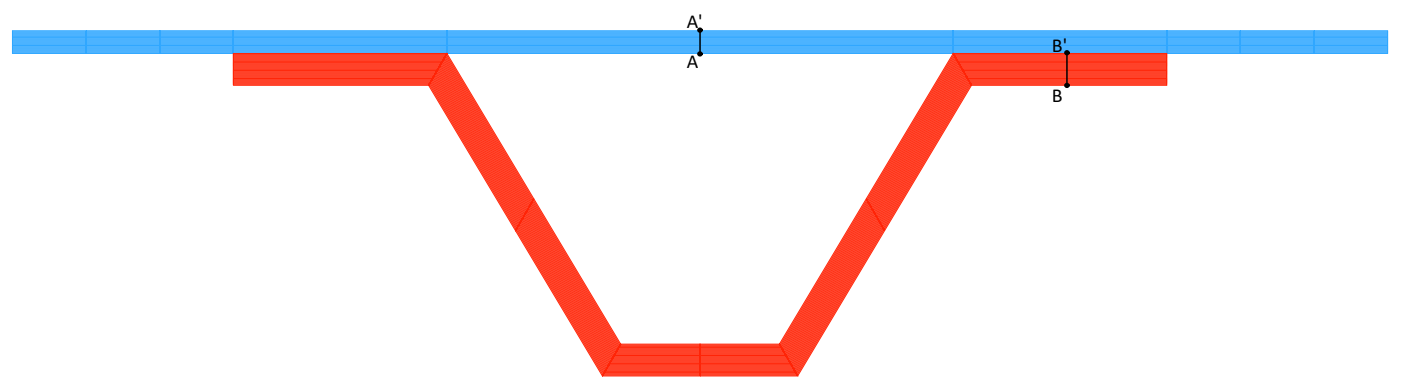

Figure 6: Cross-section of the panel RU.

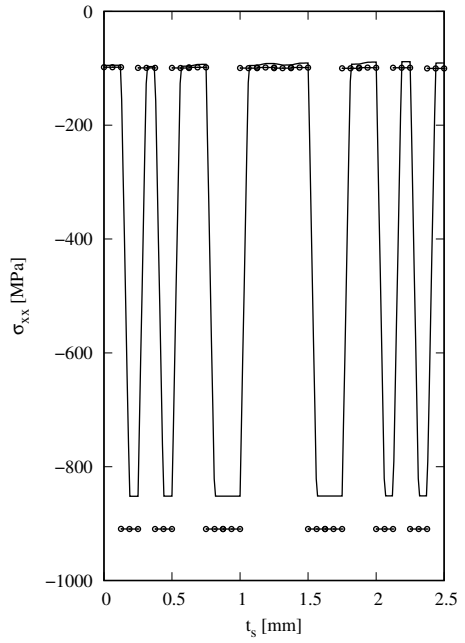

(a) Skin $\left(\mathrm{A}^{-} \mathrm{A}^{\prime}\right)-\sigma_{x x}$



(b) Skin (A-A') $\sigma_{x z}$

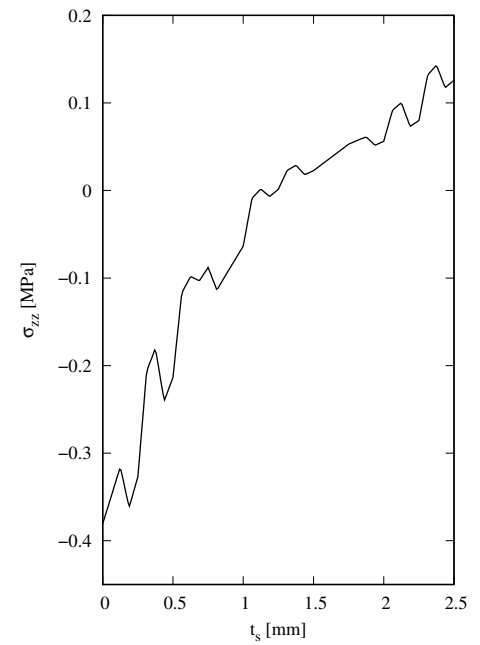

(c) Skin (A-A'). $\sigma_{z z}$

Figure 7: Mid-span distributions of stresses components through the skin thickness (A-A') of the optimum panel S1-B1; solid line "--" is CUF solution, circles "o" represent ANSYS solution.

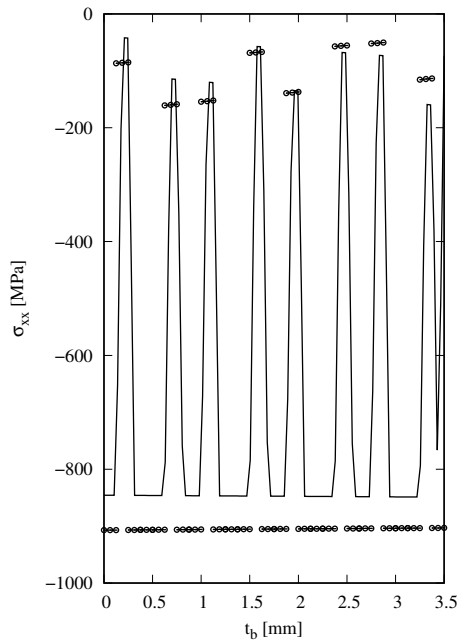

(a) Stringer (B-B'). $\sigma_{x x}$

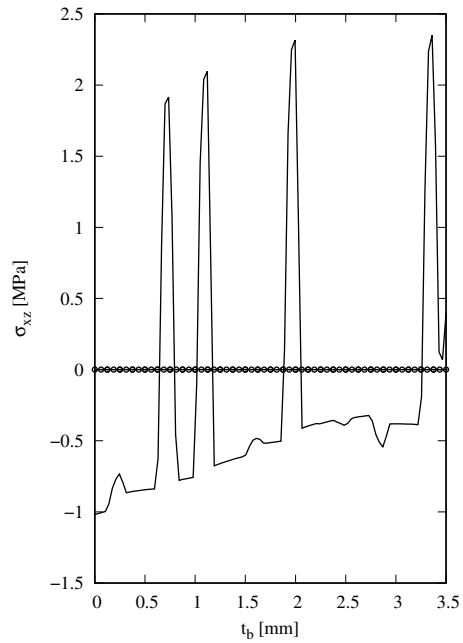

(b) Stringer (B-B'). $\sigma_{x z}$



(c) Stringer (B-B'). $\sigma_{z z}$

Figure 8: Mid-span distributions of stresses components through the stringer thickness (B-B') of the optimum panel S1-B1; solid line "--" is CUF solution, circles "०" represent ANSYS solution 